\title{
Communication \\ Superposition of $2 \omega$ and Electrostatic Field Induced Terahertz Waveforms in DC-Biased Two-Color Filament
}

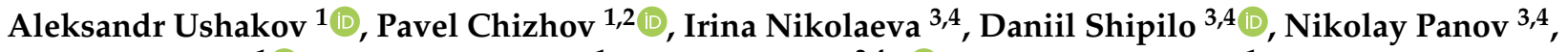 \\ Vladimir Bukin ${ }^{1}\left(\mathbb{D}\right.$, Kseniia Mamaeva ${ }^{1}$, Olga Kosareva ${ }^{3,4, *(\mathbb{D})}$ and Sergey Garnov ${ }^{1}$ \\ 1 Prokhorov General Physics Institute of the Russian Academy of Sciences, 119991 Moscow, Russia; \\ ushakov.aleksandr@physics.msu.ru (A.U.); pvch@kapella.gpi.ru (P.C.); vbkn@kapella.gpi.ru (V.B.); \\ mamaeva@physics.msu.ru (K.M.); garnov@kapella.gpi.ru (S.G.) \\ 2 Russian Institute for Scientific and Technical Information, 125315 Moscow, Russia \\ 3 Faculty of Physics, Lomonosov Moscow State University, 119991 Moscow, Russia; \\ nikolaevaia@lebedev.ru (I.N.); schipilo.daniil@physics.msu.ru (D.S.); napanov@ilc.edu.ru (N.P.) \\ 4 P.N. Lebedev Physical Institute of the Russian Academy of Sciences, 119991 Moscow, Russia \\ * Correspondence: kosareva@physics.msu.ru
}

\section{check for} updates

Citation: Ushakov, A.; Chizhov, P.; Nikolaeva, I.; Shipilo, D.; Panov, N.; Bukin, V.; Mamaeva, K.; Kosareva, O.; Garnov, S. Superposition of $2 \omega$ and Electrostatic Field Induced Terahertz Waveforms in DC-Biased Two-Color Filament. Appl. Sci. 2021, 11, 11888. https://doi.org/10.3390/ app112411888

Academic Editor: Vyacheslav A. Trofimov

Received: 11 November 2021 Accepted: 10 December 2021 Published: 14 December 2021

Publisher's Note: MDPI stays neutral with regard to jurisdictional claims in published maps and institutional affiliations.

Copyright: (c) 2021 by the authors. Licensee MDPI, Basel, Switzerland. This article is an open access article distributed under the terms and conditions of the Creative Commons Attribution (CC BY) license (https:/ / creativecommons.org/licenses/by/ $4.0 /)$.
Abstract: Increase in conversion efficiency from a femtosecond optical pump into broadband terahertz $(\mathrm{THz})$ radiation is currently an essential issue since it boosts $\mathrm{THz}$ source performance for medicine and security applications. An air-plasma based $\mathrm{THz}$ radiation from a two-color femtosecond filament is the most efficient gas-based $\mathrm{THz}$ emitter, with a dipole local source having a maximum on the beam propagation axis. In this work, we show the novel advancement to THz yield increase with preservation of the forwardly directed dipole radiation. The two-color $\mathrm{THz}$ source can be enhanced if the filament plasma channel is placed into an external electrostatic field (DC bias), which is parallel to the second harmonic polarization direction. In the experiment, we produce a plasma channel from 800-nm, 50-fs, 2-mJ pulse with $200 \mu \mathrm{J}$ of 400-nm, 50-fs mixed with the pump, and allocate it between the electrodes carrying $7-\mathrm{kV} / \mathrm{cm}$ static field. Time-domain measurements and $3 D+$ time simulations of $\mathrm{THz}$ waveforms from the two-color DC-biased filament show that the $\mathrm{THz}$ emission is the superposition of the THz waveforms generated in the $800+400-\mathrm{nm}$ filament without a DC-bias and in the $800-\mathrm{nm}$ (without $400-\mathrm{nm}$ ) plasma channel biased by $7-\mathrm{kV} / \mathrm{cm}$ static field. The additivity of the two local dipole $\mathrm{THz}$ sources is possible if the majority of free electrons are produced by the pump pulse.

Keywords: broadband $\mathrm{THz}$ pulse; methods of $\mathrm{THz}$ generation; DC-biased filament; two-color filament; interplay between $\mathrm{THz}$ local sources

\section{Introduction}

The plasma channel of a femtosecond laser filament [1-3] in air or atmospheric density gases is a promising source of terahertz $(\mathrm{THz})$ radiation [4,5]. A single-color femtosecond plasma burst [6] or a more extended filament plasma channel emits radially polarized $\mathrm{THz}$ waves, ref. [7] typically registered in the focusing geometry. Breaking a single filament symmetry through an external electric field [8] or an optical pulse at another central wavelength $[9,10]$ boosts the THz yield and paves the way to medical and security applications of THz spectroscopy [11,12].

Hereafter, we will refer to these two schemes of enhanced (as compared with a single-color plasma channel case) THz yield as the DC-biased $(\omega+D C)$ and two-color filamentation, respectively. Linearly polarized $\mathrm{THz}$ radiation is typically detected from both the DC-biased $(\omega+D C)$ and the two-color filaments [13,14]. Mixing of the pump pulse with its second harmonic, also known as the two-color $\omega+2 \omega$ filamentation, is recognised as one of the most efficient ways to generate $\mathrm{THz}$ from femtosecond gas plasmas $[15,16]$. For the mid-infrared air-based $\omega+2 \omega$ filament, the conversion efficiency to $\mathrm{THz}$ range reaches several percents [17-20]. 
The interplay between different mechanisms of $\mathrm{THz}$ generation in the filament has been studied for more than ten years. The interference in spatial domain between the radially and linearly polarized $\mathrm{THz}$ emission from DC-biased filament was reported in $[13,21,22]$ for an external field of about $1 \mathrm{kV} / \mathrm{cm}$. For the electric field strength of $5 \mathrm{kV} / \mathrm{cm}$ and larger, the linearly polarized $\mathrm{THz}$ emission dominates. The competition between the mechanisms of $\mathrm{THz}$ generation in DC-biased filament was recently experimentally studied in Ref. [23]. The analysis of THz spectra measured by electro-optical sampling shows that the balance between the sources of radially and linearly polarized $\mathrm{THz}$ emission occurs for the electrostatic field of $300 \mathrm{~V} / \mathrm{cm}$ [23].

The joint effect of the external DC bias and the second harmonic on THz yield, or, in other words, THz generation from a DC-biased two-color $(\omega+2 \omega+D C)$ filament, has been experimentally studied in Ref. [15]. In the case of an external electrostatic field orthogonal to the $\omega$ pulse polarization, Wang et al. [15] suggested that the THz emission could be the sum of two components: one generated by two-color $(\omega+2 \omega)$ laser-induced filamentation and the second induced by the DC electric field. To provide a well-controlled comparison of $\mathrm{THz}$ signal from $\omega+2 \omega$ and $\omega+D C$ filaments, Thomson et al. [24] superimposed the THz waveforms measured for $\omega+2 \omega+D C$ filament with opposite polarities to the DC-bias, assuming that $\mathrm{THz}$ signals produced with and without external electrostatic field are added coherently. This method suggests that half a sum of two THz waveforms, obtained for the opposite directions of the external electrostatic field, highlights the contribution from the $\omega+2 \omega$ source. In Ref. [25] the $\omega+2 \omega+D C$ filament was shown to be a promising tool for $\mathrm{THz}$ generation with tunable spectrum. Besides, control of the $\mathrm{THz}$ spectrum width and modulation is important for the "false" absorption frequency identification when performning $\mathrm{THz}$ spectroscopy, especially when the reflection from the layered structures is considered [12]. In atmospheric air, the tunability of the THz spectrum from a DCbiased plasma channel cannot be large, because the DC field is limited by static breakdown threshold. However, this method can be improved by considering gases with variable density or even liquids. Indeed, impressive conversion from $\omega+2 \omega$ to $\mathrm{THz}$ radiation was found in the insulator liquids [26], which have a higher breakdown threshold, thus providing a lot more freedom for spectral shaping using DC bias.

In this work, we experimentally study the THz emission from the DC-biased two-color $(\omega+2 \omega+D C)$ filament with the DC field parallel to polarization of the second harmonic and orthogonal to the fundamental light field polarization. In this case, the THz emission of both $\omega+D C$ and $\omega+2 \omega$ sources is polarized along the DC field. Terahertz waveforms measured in the temporal domain are found to be the superposition of electromagnetic fields emitted by the air-based plasma in the presence of the second harmonic pulse and the DC bias. Numerical $(t, r)+z$ simulations based on the Unidirectional Pulse Propagation Equation (UPPE, [27]) and performed separately for $\omega+D C, \omega+2 \omega$ and $\omega+2 \omega+D C$ sources in the conditions of the experiment reveal that biasing the two-color pulse with the $\mathrm{DC}$ field results in linear addition (superposition) of the THz waveforms.

The paper is organized as follows. Section 2 describes the experimental setup capable of switching the electrostatic field on and off. In the same Section 2, the 3D+time numerical approach to propagate the light field in air and to obtain the $\mathrm{THz}$ waveforms self-consistently from the simulations is presented. In Section 3, the measured and simulated waveforms from $\omega+2 \omega, \omega+D C$ and $\omega+2 \omega+D C$ are compared and discussed. Section 4 concludes the paper.

\section{Materials and Methods}

\subsection{Experimental Setup}

Figure 1 shows the scheme of the experimental setup. Ti:Sapphire laser system Spectra Physics Spitfire Pro XP delivered the pulses with the horizontal polarization centred at a wavelength of $800 \mathrm{~nm}$ with the energy up to $2.7 \mathrm{~mJ}$, for a duration of $50 \mathrm{fs}$ (full width at half maximum, FWHM) and a beam diameter of $12 \mathrm{~mm}$ (at $e^{-2}$ level) at 1-kHz repetition rate. The pulse energy was reduced down to $2 \mathrm{~mJ}$ by the half-wave plate and the Glan- 
Taylor prism. The beam was focused by the lens with a focal length of $300 \mathrm{~mm}$ forming a $\sim 15$-mm filament in free air (humidity of $30 \%$ at $23{ }^{\circ} \mathrm{C}$ ). Two copper $40 \times 15 \mathrm{~mm}^{2}$ plane electrodes with a gap of $10 \mathrm{~mm}$ between them were set parallel to the optical table so that the filament was burnt in the center between them in the transverse direction and closer to the registration system in the longitudinal direction in order to avoid the reflection of $\mathrm{THz}$ radiation from the electrodes surface. A voltage of $U_{D C}=7 \mathrm{kV}$ applied to the electrodes formed a vertically directed field with a strength of $E_{D C}=7 \mathrm{kV} / \mathrm{cm}$. To generate the second harmonic and form a two-color filament, the $\beta$-barium borate (BBO) crystal (I-type, $100-\mu \mathrm{m}$ thickness, $10 \%$ efficiency) was inserted into the pump beam after the lens. The $\mathrm{BBO}$ crystal was adjusted to maximize the second harmonic yield, so, the 400-nm radiation has the vertical polarization, i.e., perpendicular to the 800-nm pump and parallel to the external DC bias. For single-color experiments, the BBO crystal was removed from the optical path. Thus, our experimental setup allowed us to study the $\mathrm{THz}$ waveforms in three cases:
(a) $=\omega+D C$ :
DC-biased single-color filament, when BBO crystal was removed
(b) $=\omega+2 \omega$ : from the optical path;
(c) $=\omega+2 \omega+D C$ :
two-color filament, when the voltage on the electrodes was turned off $\left(U_{D C}=0\right)$;

In all cases, the $\mathrm{THz}$ radiation had linear vertical polarization. Thus, in our setup the superposition of $\mathrm{THz}$ waveforms generated according to $\omega+D C$ and $\omega+2 \omega$ mechanisms could be observed.

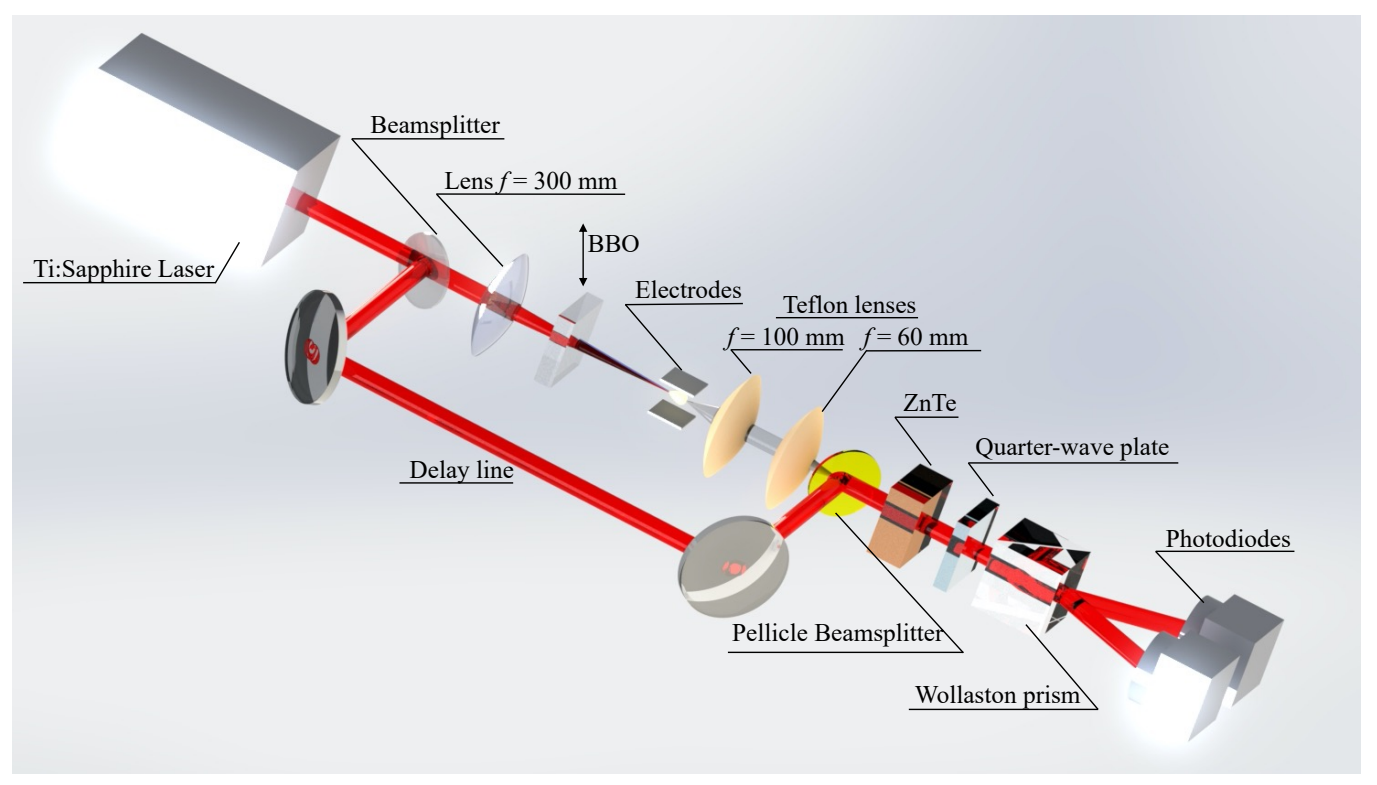

Figure 1. Scheme of the experimental setup.

For our purposes, a coherent $\mathrm{THz}$ detection technique is required. Among the three widely used methods, namely, photoconductive antenna detection [28,29], THz Air Breakdown Coherent Detection (THz-ABCD) [30], and electro-optical sampling (EOS) [31], the latter is best suited for our study. Indeed, the photoconductive antennas are regularly employed with $\mathrm{MHz}$ repetition rate laser systems, and would not have enough sensitivity for the $\mathrm{kHz}$ source we have. ABCD works very well with the two-color scheme, while the DC-biased filament produces $\mathrm{THz}$ wave with lower frequency, which is not properly detected by THz-ABCD setups. Furthermore, the THz-ABCD method requires a highpower probe pulse and an additional DC supply, which can produce distortions for the one used to bias the filament (and vice versa). In turn, EOS has excellent sensitivity, and the frequencies below $3 \mathrm{THz}$ are present in both two-color- and DC-bias-generated THz pulses. To obtain waveforms of the $\mathrm{THz}$ electric field, we used a standard time-domain system 
(TDS), which consists of the ZnTe crystal $\left(10 \times 10 \times 0.5 \mathrm{~mm}^{3},\langle 110\rangle\right.$ cut $)$, the quarter-wave plate, the Glan-Taylor prism, and two photodiodes (Figure 1). The THz field induces birefringence in ZnTe crystal, so the probe pulse attains ellipticity proportional to the field strength. After passing through the quarter-wave plate, the probe pulse is divided by the Glan-Taylor prism and is registered by the photodiodes. The difference in the photodiode signals characterizes the birefringence induced in the crystal and, therefore, the $\mathrm{THz}$ field strength. By scanning the delay between the optical pump generating the $\mathrm{THz}$ wave and the probe pulse, we obtain a THz waveform. This TDS is sensitive to the radiation in the spectral range from $\sim 0.2$ to $\sim 3 \mathrm{THz}$. Two $50-\mathrm{mm}$ diameter Teflon lenses with focal distances of 100 and $60 \mathrm{~mm}$ collimated the $\mathrm{THz}$ emission from the filament and focused it onto the ZnTe crystal. Weak THz radiation of unbiased single-color filament [6] was below the registration threshold of our $\mathrm{THz}$ detection system. However, either the $7-\mathrm{kV} / \mathrm{cm}$ static field or the second harmonic with $\sim 200 \mu \mathrm{J}$ of energy increased the THz yield up to the level of detection by TDS.

All the optical elements presented in Figure 1 were fastened and preserved their positions during the experiment. Only the BBO crystal was removed from the optical path in the case of $\omega+D C$ (a), and the mirrors of the delay line were moved. Therefore, the collection angles of $\mathrm{THz}$ radiation were the same throughout the experiment, and we can compare the measured waveforms in the three cases: $\omega+D C, \omega+2 \omega$ and $\omega+2 \omega+D C$.

\subsection{Simulations}

Filamentation and $\mathrm{THz}$ generation in the conditions of our experiment (moderate focusing with a numerical aperture of $\sim 0.02$ ) can be simulated using Unidirectional Pulse Propagation Equation (UPPE, [27]). The spatio-temporal spectrum $\widehat{\mathcal{E}}\left(\omega, k_{r}, z\right)=$ $\mathcal{F}[\mathcal{E}(t, r, z)]$ represents the electric field $\mathcal{E}(t, r, z)$ of the laser pulse together with all the secondary emission (emerging terahertz and optical harmonics). Here, $\mathcal{F}$ denotes a FourierHankel transformation from the spatio-temporal domain $(t, r, z)$ to the frequency-angular domain $\left(\omega, k_{r}, z\right)$. Although the most rigorous description of a nonlinear source term requires the solution of the Schrödinger [32] or Maxwell-Bloch Equations [33], the decomposition of the medium susceptibility into the response of bound and free electrons (or intra- and inter-band responses) is successful for $\mathrm{THz}$ generation simulations in gases [34] and transparent condensed media [26], and can even be applied to metals [35]. To decrease the computational costs, we follow this simplified approach. The external electrostatic field $E_{D C}$ is introduced into UPPE as the nonlinear susceptibility modification of the medium [36]. With this modification the third-order polarization is given by:

$$
P(t)=\chi^{(3)}\left[\left(\mathcal{E}(t)+E_{D C}\right)^{3}-E_{D C}^{3}\right],
$$

where $\chi^{(3)}$ is the third-order susceptibility of air. The transient photocurrent obeys the equation

$$
\frac{\partial J_{e}}{\partial t}=\frac{e^{2}}{m_{e}} N_{e}(t)\left(\mathcal{E}(t)+E_{D C}\right)-v_{c} J_{e}
$$

where $e$ and $m_{e}$ are electron charge and mass, respectively, $v_{c}=5 \mathrm{ps}^{-1}$ is the collision rate. The absorption current $J_{a}$ [37] does not require modification because the influence of the external electrostatic field on the plasma density $N_{e}$ is negligible. The density of free electrons $N_{e}$ was calculated from the rate equations for the major air components: molecular nitrogen $(78 \%)$ and molecular oxygen $(22 \%)$.

The nonlinear terms are calculated in time domain and substituted into UPPE:

$$
\left(\frac{\partial}{\partial z}+i k_{z}\right) \widehat{\mathcal{E}}=-\frac{2 \pi i}{c^{2} k_{z}}\left(\omega^{2} \mathcal{F}[P]-\mathcal{F}\left[\frac{\partial J_{e}}{\partial t}\right]-i \omega \mathcal{F}\left[J_{a}\right]\right)
$$


where $k_{z}=\sqrt{n^{2}(\omega) \omega^{2} / c^{2}-k_{r}^{2}}, n(\omega)$ is the refractive index of dry air, and $c$ is the speed of light. The derivative $\partial J_{e} / \partial t$ is used as it vanishes at the right boundary of the temporal domain $t \rightarrow+\infty$, whereas the photocurrent $J_{e}$ does not.

The simulations of THz generation from femtosecond filament were carried out in the experimental conditions for 800-nm pump pulse with the energy of $2 \mathrm{~mJ}$, FWHM duration of $50 \mathrm{fs}$ and beam diameter of $1 \mathrm{~cm}$. We simulated three different $\mathrm{THz}$ sources implemented in three different experimental schemes designed in our lab. These schemes are mixing the pump and electrostatic field $\omega+D C$, the pump and its second harmonic $\omega+2 \omega$, and the pump and the second harmonic in the presence of an electrostatic field $\omega+2 \omega+D C$. For the $\omega+D C$ scheme, the THz source was a single-color filament biased by the external electrostatic field $E_{D C}=7 \mathrm{kV} / \mathrm{cm}$. The $\omega+2 \omega$ case corresponds to the two-color filament formed by mixing the 800-nm pump pulse with its second harmonic (wavelength of $400 \mathrm{~nm}$, energy of $200 \mu \mathrm{J}$, FWHM duration of $35 \mathrm{fs}$ and the same beam shape as the pump). The $\omega+2 \omega+D C$ case was a combination of the previous two schemes, i.e., we simulated $\mathrm{THz}$ generation in the DC-biased two-color filament in which the second harmonic and the external field $E_{D C}$ were the same in value and direction as in the $\omega+2 \omega$ and $\omega+D C$ cases. To reproduce geometrical focusing with a focal length $f=30 \mathrm{~cm}$, we multiplied the initial field by the phase factor $\exp \left[i \omega r^{2} /(2 c f)\right]$ in $(\omega, r, z)$ domain. We propagated the pulse linearly from the lens at $z=0$ to the front edge of the electrodes at $z_{0}=26 \mathrm{~cm}$ (as in [38]) in order to decrease the computational cost. Starting from $z_{0}$ the nonlinear Equation (3) was solved.

Since the spectrum of $\mathrm{THz}$ radiation from DC-biased single-color filament covers the range up to a few terahertz $[8,13,39]$, the simulations require the spectral resolution of the order of $0.01 \mathrm{THz}$. To reduce the computational resources, we utilized the nonuniform frequency grid, which consists of two equidistant sub-grids with different steps of $\sim 0.02 \mathrm{THz}$ in the range from 0 to $85 \mathrm{THz}$ and of $\sim 0.67 \mathrm{THz}$ in the remaining frequency space up to $2.75 \mathrm{PHz}$ [36].

\section{Results and Discussion}

Figure 2 shows the measured $\mathrm{THz}$ waveforms (upper row) and the spectra of $\mathrm{THz}$ radiation reconstructed from these waveforms (dots, middle row). Panels (a)-(c) in Figure 2 correspond to $\omega+D C, \omega+2 \omega$ and $\omega+2 \omega+D C$ schemes of THz generation, respectively. The simulated THz spectra are shown in Figure 2 (middle and lower row) by solid curves. In reasonable agreement between the numerical and experimental results, the spectrum of $\mathrm{THz}$ radiation generated from a DC-biased single-color $(\omega+D C)$ filament is localized at $v \lesssim 1 \mathrm{THz}$ (Figure 2a). In the experiment, strongly divergent low-frequency $(v \approx 0.1 \mathrm{THz})$ spectral components escape the Teflon lenses and do not enter the TDS detector. Owing to this effect, the maximum of the experimental spectrum was achieved at $v \approx 0.4 \mathrm{THz}$, while the simulations predicted the spectral maximum at much lower frequencies.

In the case of the $\omega+2 \omega$ filament, the experimentally obtained and simulated spectra are much broader than in the $\omega+D C$ one (cf. Figure $2 a, b)$. The spectrum reconstructed from the measured $\mathrm{THz}$ waveform is limited by the ZnTe phonon band at $v \gtrsim 3 \mathrm{THz}$ [40]. The simulated spectrum goes down very slowly and has a width of about the inverse pulse duration in agreement with the previous studies [41]. The low-frequency part of the experimentally obtained $\mathrm{THz}$ spectrum qualitatively agrees with the simulated one (Figure 2b).

The spectrum of $\mathrm{THz}$ radiation generated in a DC-biased two-color filament $(\omega+$ $2 \omega+D C$, see Figure 2c) combines the characteristics of $\omega+D C$ and $\omega+2 \omega$ spectra considered above. This is clearly pronounced in the higher-frequency range $v \gtrsim 1.5 \mathrm{THz}$. The spectrum of $\mathrm{THz}$ radiation generated according to the $\omega+2 \omega+D C$ scheme extends up to $3 \mathrm{THz}$ and the spectral intensity at $2 \mathrm{THz}$ constitutes $10^{-2}$ of the spectrum maximum (Figure 2c). Without the second harmonic field, the spectral intensity at $2 \mathrm{THz}$ is of the order of $10^{-3}$ of the spectrum maximum (Figure 2a). This wide high-frequency wing in Figure $2 \mathrm{c}$ originates due to the $800-\mathrm{nm}$ pump pulse mixing with the second harmonic pulse 
as seen from the $\omega+2 \omega$ spectrum in Figure 2b. However, the direct intensity summation of the THz spectra in the $\omega+D C$ and $\omega+2 \omega$ schemes does not exactly reproduce the $\mathrm{THz}$ spectrum corresponding to the $\omega+2 \omega+D C$ scheme. We suggest that superposition of $\mathrm{THz}$ waves should be considered in the temporal domain. Therefore, we will study the partial contributions of the $\mathrm{THz}$ waveforms from the $\omega+D C$ and $\omega+2 \omega$ sources into the overall $\mathrm{THz}$ emission from the $\omega+2 \omega+D C$ filament.
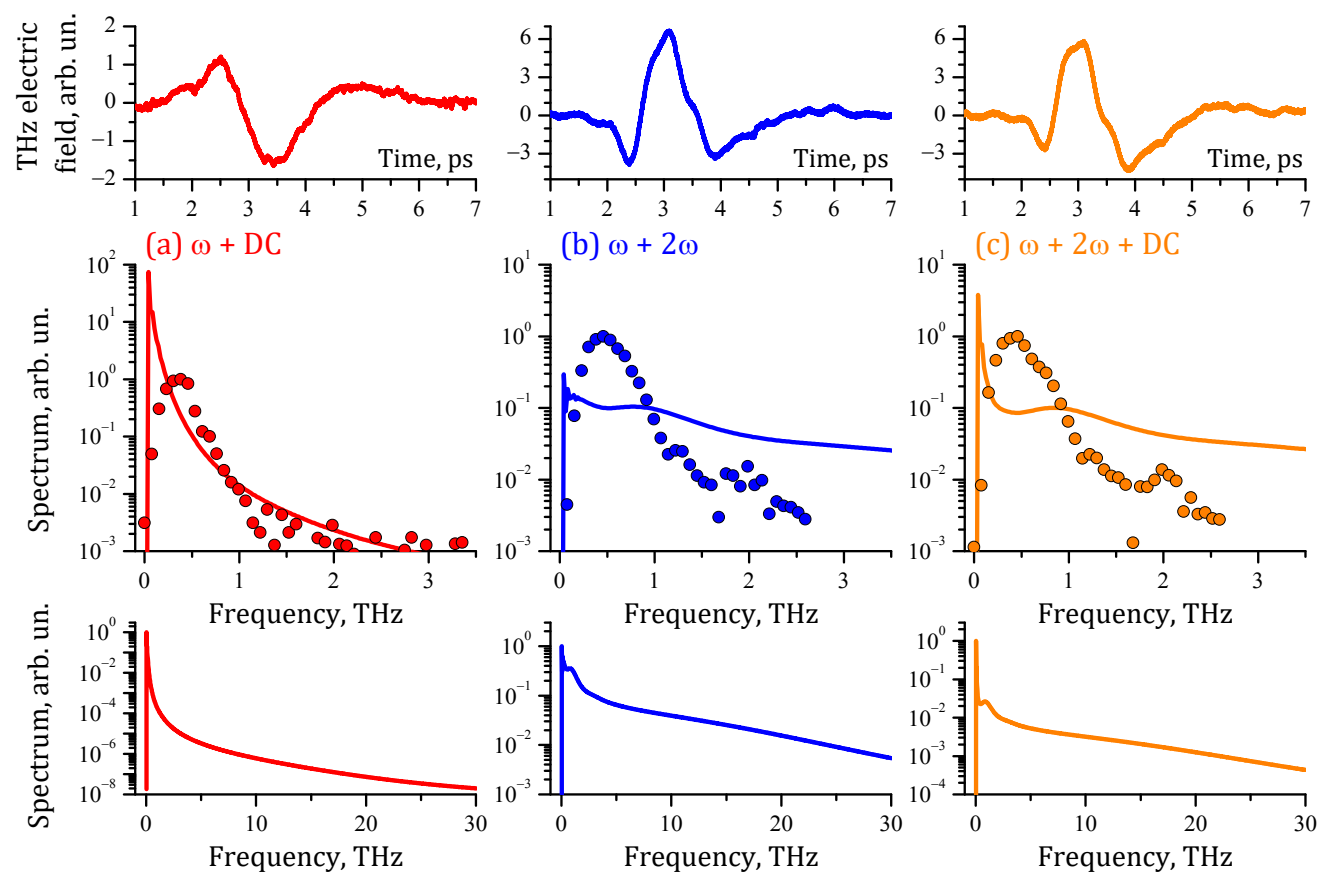

Figure 2. Upper row: measured waveforms of THz radiation emitted from ((a), red, $\omega+D C)$ DCbiased single-color filament, ((b), blue, $\omega+2 \omega)$ two-color filament and ((c), orange, $\omega+2 \omega+D C)$ DC-biased two-color filament. Middle row: THz spectra in the cases shown in upper row; (dots) $\mathrm{THz}$ spectra obtained from the measured waveforms; (solid lines) simulated THz spectra integrated over the overall transverse domain. Lower row: the simulated $\mathrm{THz}$ spectra from the middle row in the extended frequency range till $v=30 \mathrm{THz}$ and normalized.

As all the experimentally obtained waveforms were measured in the same conditions, they have the same scale and can be quantitatively compared with each other, see Figure 3a. The simulated on-axis waveforms processed by the bandpass filter $0.2 \mathrm{THz}<v<3 \mathrm{THz}$, which roughly reproduces the spectral sensitivity of TDS, are plotted in Figure $3 \mathrm{~b}$. The rectangular filter cannot fully reproduce the TDS transfer function and spatial convolution with the probe beam, so the simulated waveforms in Figure $3 \mathrm{~b}$ reproduce the measured ones in Figure 3a qualitatively. This reproduction includes the main physical characteristics of the waveforms, such as the correct relation between the amplitudes, the frequency content and the temporal shape of waveforms obtained from the $\omega+D C, \omega+2 \omega$ and $\omega+2 \omega+D C$ sources (Figure 3a,b, cf. the curves with the same colors). Note, in particular, the shorter period of $\mathrm{THz}$ field oscillations as the $2 \omega$ pulse is added to the filament (cf. red and blue curves in Figure 3a,b).

To clarify the origin of the $\mathrm{THz}$ radiation from the two-color filament placed into the electrostatic field, we subtract the $\omega+2 \omega$ waveform, obtained without the DC field, from the $\omega+2 \omega+D C$ waveform. This subtraction was performed for both measured and simulated waveforms (Figure 3c,d, black curves). As the next step, we plot the measured $\mathrm{THz}$ waveform, obtained without the second harmonic pulse, i.e., in $\omega+D C$ case, on the same panel as the result of subtraction (Figure 3c, cf. red and black curves). Similar comparison was done for the simulated $\omega+D C$ curve (Figure $3 \mathrm{~d}$, cf. red and black curves). In both the experiment and the simulations the difference between the result of $\omega+2 \omega$ 
waveform subtraction from the $\omega+2 \omega+D C$ waveform and the $\omega+D C$ waveform is $\sim 8 \%$. Thus, the THz field from a two-color filament placed into the electrostatic field is the superposition of $\mathrm{THz}$ fields from the two-color dipole source and DC-biased filament plasma dipole source.
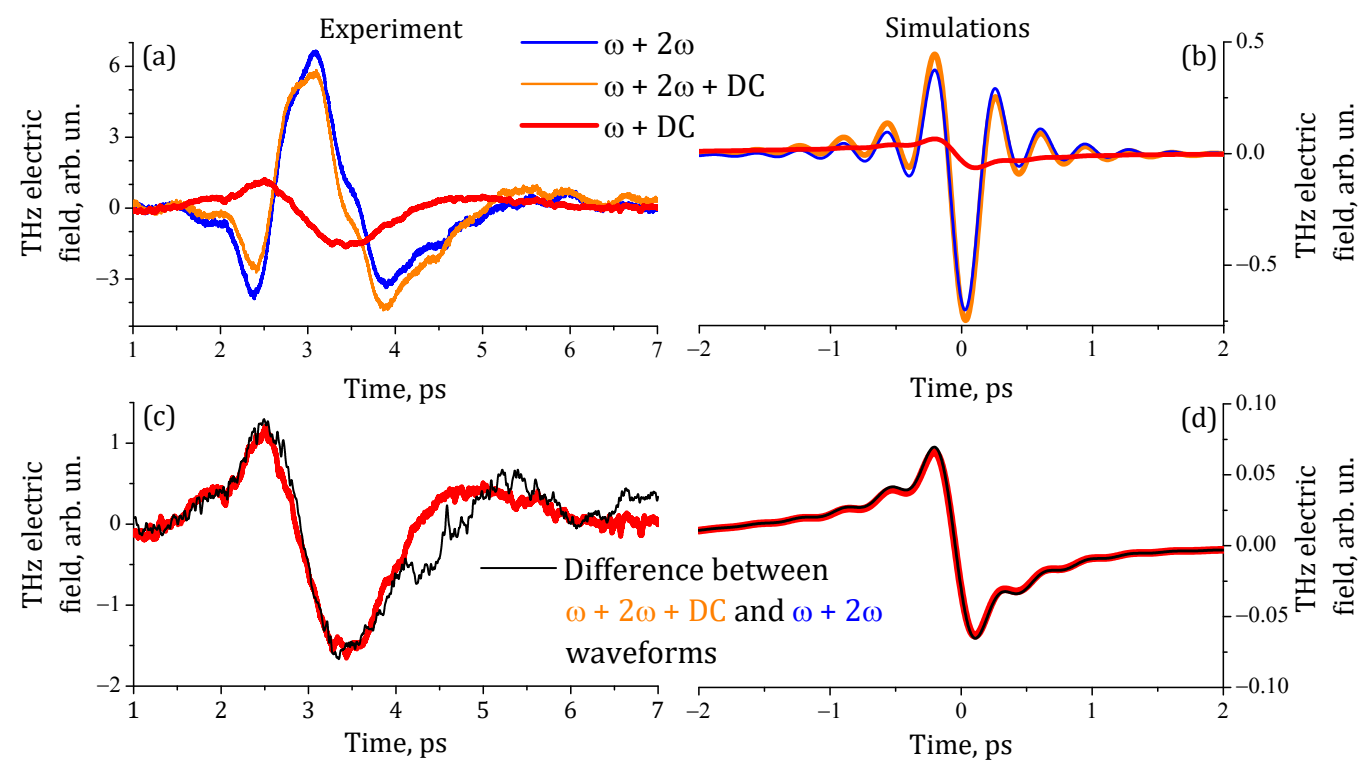

Figure 3. (a) Measured and (b) simulated waveforms of $\mathrm{THz}$ radiation generated from a two-color filament $(\omega+2 \omega$, blue curves), a DC-biased single-color filament $(\omega+D C$, red) and a DC-biased two-color filament $(\omega+2 \omega+D C$, orange). (c,d) Replica of $\omega+D C$ waveform (red) compared to the difference between $\omega+2 \omega+D C$ and $\omega+2 \omega$ waveforms (black is orange minus blue) in (c) the experiment and (d) simulations.

To confirm our proposition, let us perform the analysis based on the material equations for the atmospheric density gas ionized by a femtosecond pulse. The main source of $\mathrm{THz}$ radiation in this underdense plasma is transient photocurrent $J_{e}$ [42]. Let the laser field $\mathcal{E}(t)$ consist of the fast oscillating components only: the 800-nm pump and its second harmonic, i.e., $\mathcal{E}(t)=\mathcal{E}_{\omega}(t)+\mathcal{E}_{2 \omega}(t)$. The part of the transient photocurrent $J_{\omega}$ responsible for the generation of odd harmonics satisfies Equation (2) with $E_{D C}=0$ and $\mathcal{E}(t)=\mathcal{E}_{\omega}(t)$. The remaining part of the transient photocurrent $J_{e}-J_{\omega}$ provides for the generation of $\mathrm{THz}$ radiation (and other even optical harmonics). For $\omega+D C, \omega+2 \omega$ and $\omega+2 \omega+D C$ mechanisms of $\mathrm{THz}$ generation in the filament, the equations for the remaining part of the photocurrent following from Equation (2) are, respectively:

$$
\begin{aligned}
\frac{\partial J_{D C}}{\partial t} & =\frac{e^{2}}{m_{e}} N_{e}(t) E_{D C}-v_{c} J_{D C}, \\
\frac{\partial J_{2 \omega}}{\partial t} & =\frac{e^{2}}{m_{e}} N_{e}(t) \mathcal{E}_{2 \omega}(t)-v_{c} J_{2 \omega}, \\
\frac{\partial J_{2 \omega+D C}}{\partial t} & =\frac{e^{2}}{m_{e}} N_{e}(t)\left[\mathcal{E}_{2 \omega}(t)+E_{D C}\right]-v_{c} J_{2 \omega+D C} .
\end{aligned}
$$

Plasma density $N_{e}$ does not depend significantly on either the external DC field $E_{D C}$ or on the electric field of low-intensity second harmonic $\mathcal{E}_{2 \omega}$ (cf. red and blue curves in Figure 4). Therefore, Equation (4c) is the sum of Equations (4a) and (4b), so, $J_{2 \omega+D C}=$ $J_{D C}+J_{2 \omega}$, i.e., the THz source terms in DC-biased two-color $(\omega+2 \omega+D C)$ filament are additive. According to Equation (3), the additivity of $\mathrm{THz}$ source terms corresponds to the interference of the $\mathrm{THz}$ fields induced by them and the superposition of the measured and simulated $\mathrm{THz}$ waveforms. 

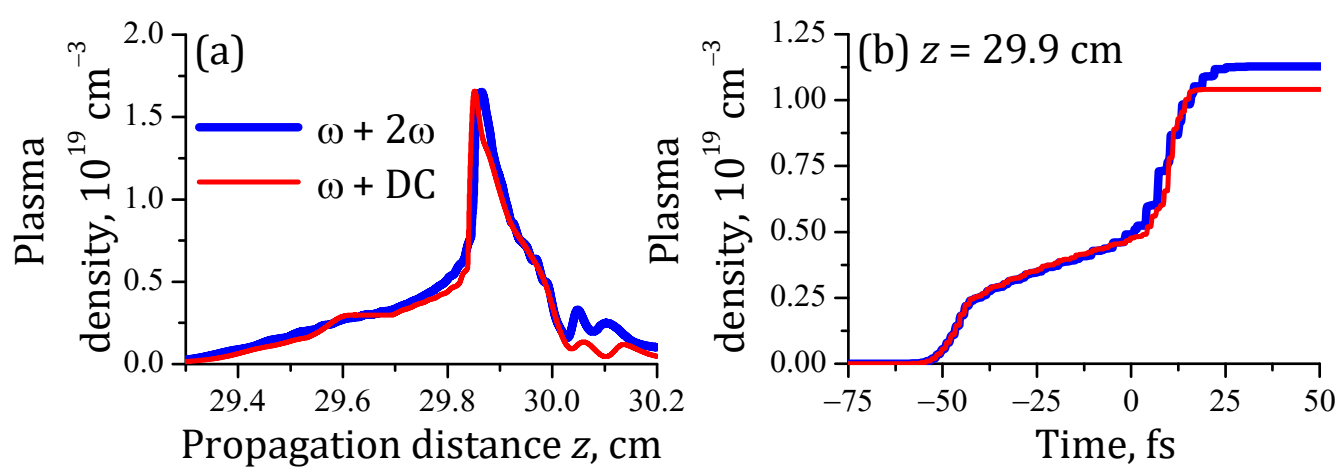

Figure 4. Dependence of maximal plasma density on (a) propagation distance $z$ and (b) local time at $z=29.9 \mathrm{~cm}$ in case of two-color filament $(\omega+2 \omega$, blue curves) and DC-biased single-color filament $(\omega+D C$, red curves).

\section{Conclusions}

In conclusion, we have shown experimentally and numerically that $\mathrm{THz}$ radiation from a two-color filament can be further enhanced by imposing the electrostatic field over the plasma channel with the direction parallel to the second harmonic pulse polarization. The physical reason for $\mathrm{THz}$ radiation enhancement is superposition of $\mathrm{THz}$ waveforms produced in the two-color filament and in the plasma channel biased by a DC electric field. The additivity of the two local dipole THz sources is possible if the majority of free electrons in the filament plasma channel is produced by the optical field of the fundamental harmonic. The superposition of $\mathrm{THz}$ waveforms observed by us could serve as a tool for $\mathrm{THz}$ pulse shaping in a DC-biased two-color filament, especially in the media with higher breakdown voltage (pressurized gases or liquids).

Author Contributions: A.U., P.C., K.M. and V.B. performed experiment and data processing; I.N., D.S., N.P. and O.K. performed data analysis and numerical simulations; V.B., O.K. and S.G. supervised the whole study. All authors have read and agreed to the published version of the manuscript.

Funding: Russian Science Foundation (grant 21-49-00023), and National Natural Science Foundation of China (grant 12061131010). A.U. thanks Presidential Scholarships for Young Scientists and Postgraduate Students (SP-500.2019.4).

Institutional Review Board Statement: Not applicable.

Informed Consent Statement: Not applicable.

Data Availability Statement: Data underlying the results presented in this paper are not publicly available at this time but may be obtained from the authors upon reasonable request.

Conflicts of Interest: The authors declare no conflict of interest.

\section{References}

1. Liu, W.; Chin, S.L. Direct measurement of the critical power of femtosecond Ti: Sapphire laser pulse in air. Opt. Express 2005, 13, 5750-5755. [CrossRef] [PubMed]

2. Couairon, A.; Mysyrowicz, A. Femtosecond filamentation in transparent media. Phys. Rep. 2007, 441, 47-189. [CrossRef]

3. Bergé, L.; Skupin, S.; Nuter, R.; Kasparian, J.; Wolf, J.P. Ultrashort filaments of light in weakly ionized, optically transparent media. Rep. Prog. Phys. 2007, 70, 1633-1713. [CrossRef]

4. Zhang, X.C.; Xu, J. Introduction to THz Wave Photonics; Springer: New York, NY, USA, 2010; Volume 29.

5. Zhang, X.C.; Shkurinov, A.; Zhang, Y. Extreme terahertz science. Nat. Photonics 2017, 11, 16. [CrossRef]

6. Hamster, H.; Sullivan, A.; Gordon, S.; White, W.; Falcone, R. Subpicosecond, electromagnetic pulses from intense laser-plasma interaction. Phys. Rev. Lett. 1993, 71, 2725. [CrossRef] [PubMed]

7. D'Amico, C.; Houard, A.; Franco, M.; Prade, B.; Mysyrowicz, A.; Couairon, A.; Tikhonchuk, V. Conical forward THz emission from femtosecond-laser-beam filamentation in air. Phys. Rev. Lett. 2007, 98, 235002. [CrossRef]

8. Löffler, T.; Jacob, F; Roskos, H. Generation of terahertz pulses by photoionization of electrically biased air. Appl. Phys. Lett. 2000, 77, 453-455. [CrossRef]

9. Cook, D.; Hochstrasser, R. Intense terahertz pulses by four-wave rectification in air. Opt. Lett. 2000, 25, 1210-1212. [CrossRef] 
10. Zhang, L.L.; Wang, W.M.; Wu, T.; Zhang, R.; Zhang, S.J.; Zhang, C.L.; Zhang, Y.; Sheng, Z.M.; Zhang, X.C. Observation of terahertz radiation via the two-color laser scheme with uncommon frequency ratios. Phys. Rev. Lett. 2017, 119, 235001. [CrossRef] [PubMed]

11. Bergé, L.; Kaltenecker, K.; Engelbrecht, S.; Nguyen, A.; Skupin, S.; Merlat, L.; Fischer, B.; Zhou, B.; Thiele, I.; Jepsen, P.U. Terahertz spectroscopy from air plasmas created by two-color femtosecond laser pulses: The ALTESSE project. Epl 2019, $126,24001$. [CrossRef]

12. Trofimov, V.A.; Wang, N.N.; Qiu, J.H.; Varentsova, S.A. Role of the pulse duration at measurements of spectral signatures of substances in $\mathrm{THz}$ range of frequencies. In Proceedings of the Millimetre Wave and Terahertz Sensors and Technology XII, Strasbourg, France, 9-12 September 2019; SPIE, the International Society for Optics and Photonics: Bellingham, WA, USA, 2019; Volume 11164, p. 1116405.

13. Houard, A.; Liu, Y.; Prade, B.; Tikhonchuk, V.T.; Mysyrowicz, A. Strong enhancement of terahertz radiation from laser filaments in air by a static electric field. Phys. Rev. Lett. 2008, 100, 255006. [CrossRef] [PubMed]

14. Kosareva, O.; Esaulkov, M.; Panov, N.; Andreeva, V.; Shipilo, D.; Solyankin, P.; Demircan, A.; Babushkin, I.; Makarov, V.; Morgner U.; et al. Polarization control of terahertz radiation from two-color femtosecond gas breakdown plasma. Opt. Lett. 2018, 43, 90-93. [CrossRef]

15. Wang, T.J.; Marceau, C.; Chen, Y.; Yuan, S.; Théberge, F.; Châteauneuf, M.; Dubois, J.; Chin, S.L. Terahertz emission from a dc-biased two-color femtosecond laser-induced filament in air. Appl. Phys. Lett. 2010, 96, 211113. [CrossRef]

16. Nikolaeva, I.; Shipilo, D.; Panov, N.; Kosareva, O. Dual-wavelength filamentation with a fraction of fundamental laser frequency as a wideband THz source. Laser Phys. Lett. 2021, 18, 025401. [CrossRef]

17. Clerici, M.; Peccianti, M.; Schmidt, B.E.; Caspani, L.; Shalaby, M.; Giguere, M.; Lotti, A.; Couairon, A.; Légaré, F.; Ozaki, T.; et al. Wavelength scaling of terahertz generation by gas ionization. Phys. Rev. Lett. 2013, 110, 253901. [CrossRef]

18. Jang, D.; Schwartz, R.M.; Woodbury, D.; Griff-McMahon, J.; Younis, A.H.; Milchberg, H.M.; Kim, K.Y. Efficient terahertz and Brunel harmonic generation from air plasma via mid-infrared coherent control. Optica 2019, 6, 1338. [CrossRef]

19. Mitrofanov, A.; Sidorov-Biryukov, D.; Nazarov, M.; Voronin, A.; Rozhko, M.; Shutov, A.; Ryabchuk, S.; Serebryannikov, E.; Fedotov, A.; Zheltikov, A. Ultraviolet-to-millimeter-band supercontinua driven by ultrashort mid-infrared laser pulses. Optica 2020, 7, 15. [CrossRef]

20. Koulouklidis, A.D.; Gollner, C.; Shumakova, V.; Fedorov, V.Y.; Pugžlys, A.; Baltuška, A.; Tzortzakis, S. Observation of extremely efficient terahertz generation from mid-infrared two-color laser filaments. Nat. Commun. 2020, 11, 1. [CrossRef]

21. Akhmedzhanov, R.; Ilyakov, I.; Mironov, V.; Suvorov, E.; Fadeev, D.; Shishkin, B. Plasma mechanisms of pulsed terahertz radiation generation. Radiophys. Quantum Electron. 2009, 52, 482-493. [CrossRef]

22. Suvorov, E.; Akhmedzhanov, R.; Fadeev, D.; Ilyakov, I.; Mironov, V.; Shishkin, B. On the peculiarities of THz radiation generation in a laser induced plasmas. J. Infrared Millim. Terahertz Waves 2011, 32, 1243-1252. [CrossRef]

23. Feng, S.; Dong, L.; Tan, Y.; Wu, T.; Ma, D.; Zhang, L.; Zhang, C.; Zhao, Y. Observation on the competition mechanism of terahertz wave generation from filament in bias electric field. Opt. Commun. 2020, 473, 125917. [CrossRef]

24. Thomson, M.D.; Kreß, M.; Löffler, T.; Roskos, H.G. Broadband THz emission from gas plasmas induced by femtosecond optical pulses: From fundamentals to applications. Las. Photon. Rev. 2007, 1, 349-368.

25. Zhang, Z.; Chen, Y.; Zhang, Z.; Xia, T.; Zhang, J.; Sheng, Z. Frequency blue shift of terahertz radiation from femtosecond laser induced air plasmas. Appl. Phys. B 2021, 127, 1-5. [CrossRef]

26. Dey, I.; Jana, K.; Fedorov, V.Y.; Koulouklidis, A.D.; Mondal, A.; Shaikh, M.; Sarkar, D.; Lad, A.D.; Tzortzakis, S.; Couairon, A.; et al. Highly efficient broadband terahertz generation from ultrashort laser filamentation in liquids. Nat. Commun. 2017, 8, 1184. [CrossRef] [PubMed]

27. Kolesik, M.; Moloney, J.V. Nonlinear optical pulse propagation simulation: From Maxwell's to unidirectional equations. Phys. Rev. E 2004, 70, 036604. [CrossRef]

28. Auston, D.H.; Cheung, K.P.; Smith, P.R. Picosecond photoconducting Hertzian dipoles. Appl. Phys. Lett. 1984, 45, 284-286. [CrossRef]

29. Smith, P.R.; Auston, D.H.; Nuss, M.C. Subpicosecond photoconducting dipole antennas. IEEE J. Quantum Electron. 1988, 24, 255-260. [CrossRef]

30. Dai, J.; Xie, X.; Zhang, X.C. Detection of broadband terahertz waves with a laser-induced plasma in gases. Phys. Rev. Lett. 2006, 97, 103903. [CrossRef]

31. Wu, Q.; Zhang, X.C. Free-space electro-optic sampling of terahertz beams. Appl. Phys. Lett. 1995, 67, 3523-3525. [CrossRef]

32. Kostin, V.A.; Laryushin, I.D.; Silaev, A.A.; Vvedenskii, N.V. Ionization-Induced Multiwave Mixing: Terahertz Generation with Two-Color Laser Pulses of Various Frequency Ratios. Phys. Rev. Lett. 2016, 117, 035003. [CrossRef]

33. Lindberg, M.; Koch, S.W. Effective Bloch equations for semiconductors. Phys. Rev. B 1988, 38, 3342. [CrossRef]

34. Bergé, L.; Skupin, S.; Köhler, C.; Babushkin, I.; Herrmann, J. 3D Numerical Simulations of THz Generation by Two-Color Laser Filaments. Phys. Rev. Lett. 2013, 110, 073901. [CrossRef] [PubMed]

35. Citroni, R.; Di Paolo, F.; Di Carlo, A. Replacing noble metals with alternative metals in MID-IR frequency: A theoretical approach. In Proceedings of the AIP Conference NANOINNOVATION 2017, Rome, Italy, 26-29 September 2017; AIP Pullishing LLC: College Park, MD, USA, 2018; Volume 1990, p. 020004. 
36. Nikolaeva, I.; Shipilo, D.; Pushkarev, D.; Rizaev, G.; Mokrousova, D.; Koribut, A.; Grudtsyn, Y.; Panov, N.; Seleznev, L.; Liu, W.; et al. Flat-top THz directional diagram of a DC-biased filament. Opt. Lett. 2021, 46, 5497-5500. [CrossRef]

37. Rae, S.; Burnett, K. Detailed simulations of plasma-induced spectral blueshifting. Phys. Rev. A 1992, 46, 1084. [CrossRef]

38. Zhang, Z.; Panov, N.; Andreeva, V.; Zhang, Z.; Slepkov, A.; Shipilo, D.; Thomson, M.; Wang, T.J.; Babushkin, I.; Demircan, A.; et al. Optimum chirp for efficient terahertz generation from two-color femtosecond pulses in air. Appl. Phys. Lett. 2018, 113, 241103. [CrossRef]

39. Chen, Y.; Wang, T.j.; Marceau, C.; Théberge, F.; Châteauneuf, M.; Dubois, J.; Kosareva, O.; Chin, S.L. Characterization of terahertz emission from a dc-biased filament in air. Appl. Phys. Lett. 2009, 95, 101101. [CrossRef]

40. Gallot, G.; Zhang, J.; McGowan, R.; Jeon, T.I.; Grischkowsky, D. Measurements of the THz absorption and dispersion of ZnTe and their relevance to the electro-optic detection of THz radiation. Appl. Phys. Lett. 1999, 74, 3450-3452. [CrossRef]

41. Borodin, A.V.; Panov, N.A.; Kosareva, O.G.; Andreeva, V.A.; Esaulkov, M.N.; Makarov, V.A.; Shkurinov, A.P.; Chin, S.L.; Zhang, X.C. Transformation of terahertz spectra emitted from dual-frequency femtosecond pulse interaction in gases. Opt. Lett. 2013, 38, 1906-1908. [CrossRef]

42. Kim, K.Y.; Glownia, J.H.; Taylor, A.J.; Rodriguez, G. Terahertz emission from ultrafast ionizing air in symmetry-broken laser fields. Opt. Express 2007, 15, 4577-4584. [CrossRef] [PubMed] 